\title{
Time-domain Field Responses of the Thin, High-contrast, Finely Layered Structure in IC Packagings
}

\author{
Adrianus T. de Hoop \\ Laboratory of Electromagnetic Research, \\ Mathematics and Computer Science, \\ Delft University of Technology, \\ Mekelweg 4, 2628 CD Delft, the Netherlands \\ Email: A.T.deHoop@tudelft.nl
}

\author{
Lijun Jiang \\ The Department of Electronic and Electrical Engineering, \\ the University of Hong Kong, Hong Kong \\ IBM T. J. Watson Research Center, \\ 1101 Kitchawan Road, Yorktown Heights, NY 10598 \\ Email: ljiang@eee.hku.hk, ljiang@us.ibm.com
}

\begin{abstract}
The thin, high-contrast, fine layers with dielectric and conductive properties, such as ground planes, are feature structures in IC packagings. Their responses to the pulsed electromagnetic field is important both theoretically and practically. In this paper, a new semi-analytical method is proposed to model the interaction of the layer with an incident electromagnetic field via a boundary condition that expresses the in-plane conduction and contrast electric polarization currents in terms of the exciting incident field by relating the jump in the tangential component of the magnetic field strength across the layer in terms of the (continuous) tangential component of the electric field strength in the layer. Expressions for pulse shapes of the reflected and transmitted fields are conveniently obtained based on this method. It provides a novel angle to investigate the ground plane effects inside IC packagings.
\end{abstract}

\section{INTRODUCTION}

The impetus for the research arose in the context of the computational investigation into the signal integrity of electromagnetic fields in the layered IC structures. In the analysis, design and optimizations stages of all relevant technical applications, as well as their associated electromagnetic interference analysis, the computational modeling of the governing electromagnetic field is a subject of considerable importance and concern. Standard approaches to the discretization of Maxwell's equations in such structures are faced with serious difficulties as to the meshing or gridding of the configuration [1]. To overcome this difficulty, several approximate procedures have been proposed. They are all of a physically heuristic nature and their degree of accuracy and usefulness requires testing against the results of certain benchmark configurations .

As to the heuristic approaches one can, broadly speaking, distinguish three different kinds of reasoning. In [2], the contrast volume source type electromagnetic field representations with the Green's functions (propagators) of the homogeneous, isotropic embedding (usually free space) serve as the point of departure. By a judicious reasoning, the pertaining thin layers of contrast volume electric and magnetic current are replaced with their corresponding equivalent surface currents. In [3], the frequency-domain counterpart of the Lorentz reciprocity relation of the time-convolution type [4, Section 28.2] is alternatively applied to the interior of the layer and the exterior of the layer, under the use of field reaction concept introduced by Rumsey [5] and the field equivalence theorem [6, Chapter 30]. These two approaches are of the global type in that they employ field representations or theorems that apply to the configuration space as a whole to arrive at more or less local interface jump conditions across the layer. Such local conditions can, however, also be constructed directly from local arguments applied to Maxwell's equations. This third kind of approach is followed by Collin [7, p. 693] and by Senior [8]. A fourth kind of approach goes via the use of surface integral equations [1]. An overview of the different approaches, as well as their application to a number of canonical problems, can be found in [9].

In the present paper we employ a kind of local approach that was introduced by Schoenberg and Muir [10] in the context of elastodynamic wave propagation in finely layered rock formations and that has also been applied to acoustic wave propagation [11]. The procedure leads to local jump conditions on the tangential components of the electric and/or magnetic field strengths across the layer, the coefficients in which can be interpreted as local Kirchhoff lumped electric circuit elements (such as conductance and capacitance for a conductive and dielectric layer), whose values are representative for the field transfer in the direction normal to the layer.

Up to the present, the literature on the subject is concentrated on the frequency-domain analysis of the electromagnetic fields involved. Nowadays, a substantial range of applications employs pulsed EM fields. For this reason, we concentrate on the direct time-domain analysis of the problem and correspondingly use some auxiliary tools from linear, time-invariant, causal system's theory, amongst which the time Laplace transformation properties of causal signals. The configuration that we consider is a thin, finely layered, highcontrast, planar sheet with electrically conductive and dielectric properties that differ from the ones of the surrounding medium. Its representative structure is the ground plane in IC 


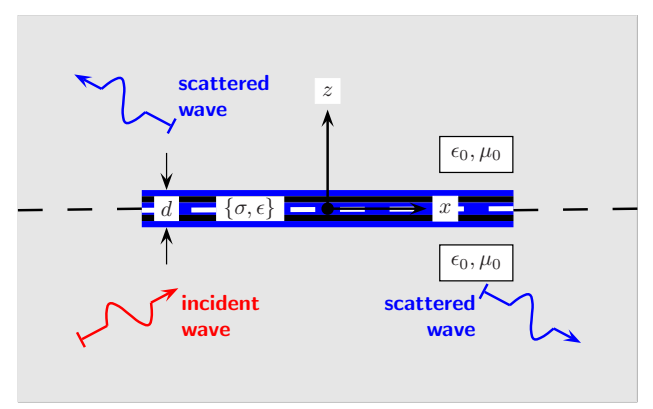

Fig. 1. Configuration with thin, finely layered, high-contrast structure in a homogeneous, isotropic background medium, with incident and scattered EM waves.

packagings.

\section{Boundary Conditions of the Thin, High CONTRAST LAYER}

The high-contrast, layered specimen occupies the domain $\mathcal{D}=\left\{(x, y) \in \Sigma \subset \mathbb{R}^{2},-d / 2<z<d / 2\right\}$, where $d>0$ is the thickness of the layer and $\Sigma$ is its spatial support in the $(x, y)$-plane. The background medium occupies the entire $\mathbb{R}^{3}$. Somewhere in this medium, sources generate an incident electromagnetic field. This field is scattered by the contrasting specimen (Figure 1). The background medium is taken to be lossless, homogeneous and isotropic with electric permittivity $\epsilon_{0}$ and magnetic permeability $\mu_{0}$. The electromagnetic properties of the layer are characterized by its electric conductivity $\sigma(\boldsymbol{r})$ and its electric permittivity $\epsilon(\boldsymbol{r})$; its magnetic permeability $\mu_{0}$ is taken to be the same as the one of the background medium.

We assume the travel time of the incident wave across the layer to be negligible with respect to the time width of the incident pulse. Furthermore, the electric properties of the layered material, i.e., its electric conductivity and/or its electric permittivity are assumed to be so high that the quantities

$$
G_{\mathrm{L}}=\int_{z=-d / 2}^{d / 2} \sigma(\boldsymbol{r}) \mathrm{d} z, \quad C_{\mathrm{L}}=\int_{z=-d / 2}^{d / 2} \epsilon(\boldsymbol{r}) \mathrm{d} z .
$$

are of order $O(1)$ as $d \downarrow 0$. (Note that $G_{\mathrm{L}}$ has the dimension of a conductance and $C_{\mathrm{L}}$ has the dimension of a capacitance.)

Using a 'pillbox' perpendicularly crossing the layer and exercising the vector Stokes' Theorem, the following boundary conditions considering the field jump over the thin layer can be derived

$$
\begin{array}{r}
\boldsymbol{i}_{z} \times \boldsymbol{E}^{\mathrm{s}}(x, y, d / 2, t)-\boldsymbol{i}_{z} \times \boldsymbol{E}^{\mathrm{s}}(x, y,-d / 2, t) \\
=O(d) \text { for }(x, y) \in \Sigma \text { as } d \downarrow 0 \\
\boldsymbol{i}_{z} \times \boldsymbol{H}^{\mathrm{s}}(x, y, d / 2, t)-\boldsymbol{i}_{z} \times \boldsymbol{H}^{\mathrm{s}}(x, y,-d / 2, t) \\
=\boldsymbol{J}_{\text {sheet }}^{\mathrm{s}}+O(d) \text { for }(x, y) \in \Sigma \text { as } d \downarrow 0 .
\end{array}
$$

where

$$
\boldsymbol{J}_{\text {sheet }}^{\mathrm{s}}=\left(G_{\mathrm{L}}+C_{\mathrm{L}} \partial_{t}\right) \boldsymbol{E}(x, y, 0, t) \text { for }(x, y) \subset \Sigma \text {. }
$$

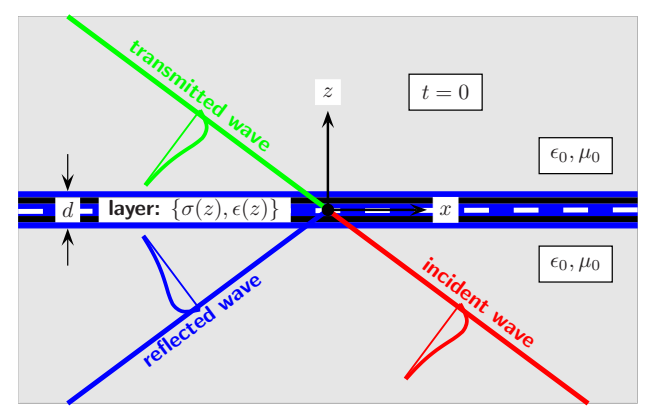

Fig. 2. Configuration with finely layered, high-contrast layer of infinite extent, with incident, reflected and transmitted pulsed EM waves.

\section{Time Domain Scattering CoefFicients}

The thin, high-contrast layer of infinite extent occupies the plane $\{z=0\}$. Define the domain $\mathcal{D}^{-}=\left\{(x, y) \in \mathbb{R}^{2},-\infty<\right.$ $z<0\}$ and $\mathcal{D}^{+}=\left\{(x, y) \in \mathbb{R}^{2}, 0<z<\infty\right\}$ to be the reflection and transmission regions around the layer (Figure $2)$. The incident wave is taken to propagate in the $(x, z)$-plane which makes all field components independent of $y$. In view of this property, the electromagnetic field in the configuration decomposes into an $E$-polarized field with the electric field parallel to the layer and an $H$-polarized field with the magnetic field parallel to the layer. Then, using the scattering description discussed in Section II, taking into account the symmetry properties and denoting by $\boldsymbol{\alpha}=\alpha_{x} \boldsymbol{i}_{x}+\alpha_{z} \boldsymbol{i}_{z}$, with $\alpha_{x}^{2}+\alpha_{z}^{2}=1$, the unit vector in the direction of propagation of the incident wave, the representations for the field components can be written as:

\section{E-polarized field:}

$$
\begin{aligned}
& \left\{H_{x}^{\mathrm{i}}, E_{y}^{\mathrm{i}}, H_{z}^{\mathrm{i}}\right\}=\left\{-\alpha_{z} Y_{0}, 1, \alpha_{x} Y_{0}\right\} \times \\
& E_{0}^{\mathrm{i}}\left[t-\left(\alpha_{x} x+\alpha_{z} z\right) / c_{0}\right] \text { for } \boldsymbol{r} \in \mathbb{R}^{3} . \\
& \left\{H_{x}^{\mathrm{s}}, E_{y}^{\mathrm{s}}, H_{z}^{\mathrm{s}}\right\}=\left\{\mp \alpha_{z} Y_{0}, 1, \alpha_{x} Y_{0}\right\} \times \\
& E_{0}^{\mathrm{s}}\left[t-\left(\alpha_{x} x \pm \alpha_{z} z\right) / c_{0}\right] \text { for } \boldsymbol{r} \in \mathcal{D}^{ \pm} .
\end{aligned}
$$

where $c_{0}=\left(\epsilon_{0} \mu_{0}\right)^{-1 / 2}$ is the electromagnetic wave speed in the background medium, $Y_{0}=\left(\epsilon_{0} / \mu_{0}\right)^{1 / 2}$ is the planewave admittance of the medium, $E_{0}^{\mathrm{i}}(t)$ is the signature of the incident $E$-polarized pulse, $E_{0}^{\mathrm{s}}(t)$ is the signature of the scattered $E$-polarized pulse, and

\section{H-polarized field:}

$$
\begin{gathered}
\left\{E_{x}^{\mathrm{i}}, H_{y}^{\mathrm{i}}, E_{z}^{\mathrm{i}}\right\}=\left\{\alpha_{z} Z_{0}, 1,-\alpha_{x} Z_{0}\right\} \times \\
H_{0}^{\mathrm{i}}\left[t-\left(\alpha_{x} x+\alpha_{z} z\right) / c_{0}\right] \text { for } \boldsymbol{r} \in \mathbb{R}^{3} . \\
\left\{E_{x}^{\mathrm{s}}, H_{y}^{\mathrm{s}}, E_{z}^{\mathrm{s}}\right\}= \pm\left\{ \pm \alpha_{z} Z_{0}, 1,-\alpha_{x} Z_{0}\right\} \times \\
H_{0}^{\mathrm{s}}\left[t-\left(\alpha_{x} x \pm \alpha_{z} z\right) / c_{0}\right] \text { for } \boldsymbol{r} \in \mathcal{D}^{ \pm} .
\end{gathered}
$$

where $Z_{0}=\left(\mu_{0} / \epsilon_{0}\right)^{1 / 2}$ is the plane-wave impedance of the medium, $H_{0}^{\mathrm{i}}(t)$ is the signature of the incident $H$-polarized 
pulse and is $H_{0}^{\mathrm{i}}(t)$ the signature of the scattered $H$-polarized pulse.

In view of the linearity and the time invariance of the configuration, we can write

$$
E_{0}^{\mathrm{s}}(t)=S^{E}(t) \stackrel{(t)}{*} E_{0}^{\mathrm{i}}(t), \quad H_{0}^{\mathrm{s}}(t)=S^{H}(t) \stackrel{(t)}{*} H_{0}^{\mathrm{i}}(t) .
$$

with $S^{E}(t)$ as the time-domain scattering coefficient of $E$ polarized waves, and with $S^{H}(t)$ as the time-domain scattering coefficient of $H$-polarized waves and where $\stackrel{(t)}{*}$ denotes time convolution.

Using the time Laplace transforms of the field representations (9) in the time Laplace transforms of the boundary conditions (2) and (3) leads to E-polarized field $\hat{S}^{E}(s)$ and H-polarized field $\hat{S}^{H}(s)$ :

$$
\begin{gathered}
\hat{S}^{E}(s)=-1+\frac{2 \alpha_{z} Y_{0}}{2 \alpha_{z} Y_{0}+G_{\mathrm{L}}+s C_{\mathrm{L}}} . \\
\hat{S}^{H}(s)=-1+\frac{2}{2+\alpha_{z} Z_{0}\left(G_{\mathrm{L}}+s C_{\mathrm{L}}\right)} .
\end{gathered}
$$

The corresponding time-domain expression for E-polarized fields is:

$$
S^{E}(t)=-\delta(t)+T^{E} \exp \left(-\beta^{E} t\right) H(t)
$$

with $T^{E}=2 \alpha_{z} Y_{0} / C_{\mathrm{L}}$ and $\beta^{E}=T^{E}+G_{\mathrm{L}} / C_{\mathrm{L}}$. The corresponding time-domain expression for $\mathrm{H}$-polarized fields is:

$$
S^{H}(t)=-\delta(t)+T^{H} \exp \left(-\beta^{H} t\right) H(t)
$$

with $T^{H}=2 / \alpha_{z} Z_{0} C_{\mathrm{L}}$ and $\beta^{H}=T^{H}+G_{\mathrm{L}} / C_{\mathrm{L}} . \delta(t)$ is the Dirac delta distribution and $H(t)$ is the Heaviside unit step function. (Note that $\beta^{E}>T^{E}$ and $\beta^{H}>T^{H}$.)

For no contrast in the layer $\left(G_{\mathrm{L}}=0\right.$ and $\left.C_{\mathrm{L}}=0\right)$, both $S^{E}(t)=0$ and $S^{H}(t)=0$, as it should (no scattering). In the special case of conductive contrast only $\left(G_{\mathrm{L}} \neq 0\right.$, $\left.C_{\mathrm{L}}=0\right), S^{E}(t)=-\left[G_{\mathrm{L}} /\left(2 \alpha_{z} Y_{0}+G_{\mathrm{L}}\right)\right] \delta(t)$ and $S^{H}(t)=$ $-\left[\alpha_{z} Z_{0} G_{\mathrm{L}} /\left(2+\alpha_{z} Z_{0} G_{\mathrm{L}}\right)\right] \delta(t)$.

\section{Numerical Results}

In this section we present some numerical results for two types of pulse shape of the incident wave: the rectangular pulse and the trapezoidal pulse. The trapezoidal pulse is the frequently used signal in the analysis of practical band limited IC interconnects and packagings. The governing electromagnetic parameters in the reflection/transmission structure are: the layer conductance ratio

$$
\eta_{\mathrm{L}}=G_{\mathrm{L}} / Y_{0}=G_{\mathrm{L}} Z_{0} .
$$

the layer admittance time constant

$$
\tau_{\mathrm{L}}=C_{\mathrm{L}} / G_{\mathrm{L}} .
$$

The incident wave is characterized by its direction of incidence $\alpha_{x}$ (projection of the unit vector along the direction of propagation on the plane of the scattering layer), its pulse time width $t_{\mathrm{w}}$ for the rectangular pulse and its pulse rise time $t_{\mathrm{r}}$,

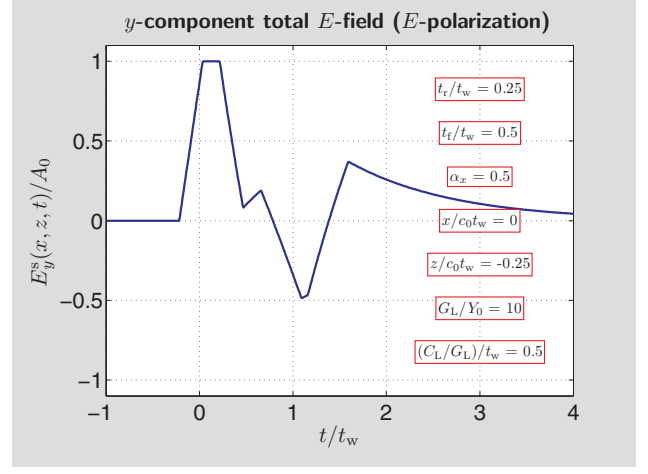

Fig. 3. Time snap in front of, and in the vicinity of, the scattering layer ( $E$-polarization, trapezoidal incident pulse).

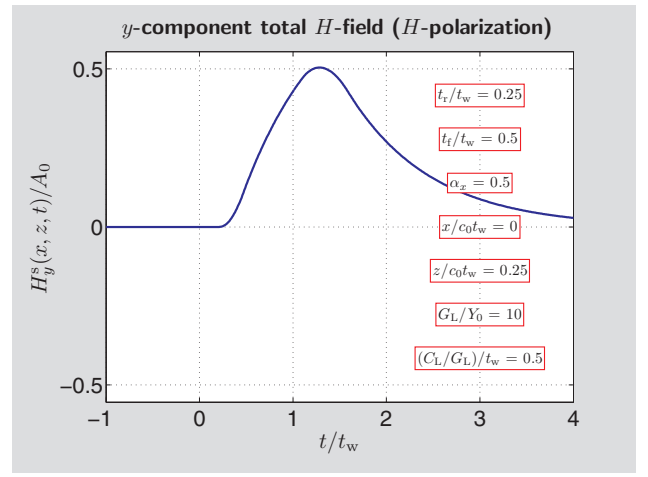

Fig. 4. Time snap behind, and in the vicinity of, the scattering layer ( $H$ polarization, trapezoidal incident pulse).

pulse time width $t_{\mathrm{w}}$ and pulse fall time $t_{\mathrm{f}}$ of the trapezoidal pulse.

For the case of the incident wave with trapezoidal pulse shape, we take, for the two types of polarization,

$$
\begin{aligned}
\left\{E_{0}^{\mathrm{i}}, H_{0}^{\mathrm{i}}\right\}= & A_{0}\left[t_{\mathrm{r}}^{-1} \operatorname{ramp}(t)-t_{\mathrm{r}}^{-1} \operatorname{ramp}\left(t-t_{\mathrm{r}}\right)-\right. \\
& t_{\mathrm{f}}^{-1} \operatorname{ramp}\left(t-t_{\mathrm{r}} / 2-t_{\mathrm{w}}+t_{\mathrm{f}} / 2\right)+ \\
& t_{\mathrm{f}}^{-1} \operatorname{ramp}\left(t-t_{\mathrm{r}} / 2-t_{\mathrm{w}}-t_{\mathrm{f}} / 2\right) .
\end{aligned}
$$

where $A_{0}$ is the pulse amplitude and $\operatorname{ramp}(t)$ is the unit ramp function $(\operatorname{ramp}(t)=\{0, t\}$ for $\{t<0, t \geq 0\}$. Here, the fundamental constituent to the pulse shape of the scattered wave is its unit ramp response, which we denote, for the two types of polarization, by $\left\{\left.e^{\mathrm{s}}\right|_{\mathrm{ramp}},\left.h^{\mathrm{s}}\right|_{\mathrm{ramp}}\right\}(t)$. Obviously, in terms of this response,

$$
\begin{aligned}
& \left\{E_{0}^{\mathrm{s}}, H_{0}^{\mathrm{s}}\right\}(t)=A_{0}\left[t_{\mathrm{r}}^{-1}\left\{\left.e^{\mathrm{s}}\right|_{\mathrm{ramp}},\left.h^{\mathrm{s}}\right|_{\mathrm{ramp}}\right\}(t)-\right. \\
& t_{\mathrm{r}}^{-1}\left\{\left.e^{\mathrm{s}}\right|_{\mathrm{ramp}},\left.h^{\mathrm{s}}\right|_{\mathrm{ramp}}\right\}\left(t-t_{\mathrm{r}}\right)- \\
& t_{\mathrm{f}}^{-1}\left\{\left.e^{\mathrm{s}}\right|_{\mathrm{ramp}},\left.h^{\mathrm{s}}\right|_{\mathrm{ramp}}\right\}\left(t-t_{\mathrm{r}} / 2-t_{\mathrm{w}}+t_{\mathrm{f}} / 2\right)+ \\
& \left.t_{\mathrm{f}}^{-1}\left\{\left.e^{\mathrm{s}}\right|_{\mathrm{ramp}},\left.h^{\mathrm{s}}\right|_{\mathrm{ramp}}\right\}\left(t-t_{\mathrm{r}} / 2-t_{\mathrm{w}}-t_{\mathrm{f}} / 2\right)\right] .
\end{aligned}
$$

\section{Unit ramp response (E-polarization)}

On account of the results of Section III, the time Laplace 


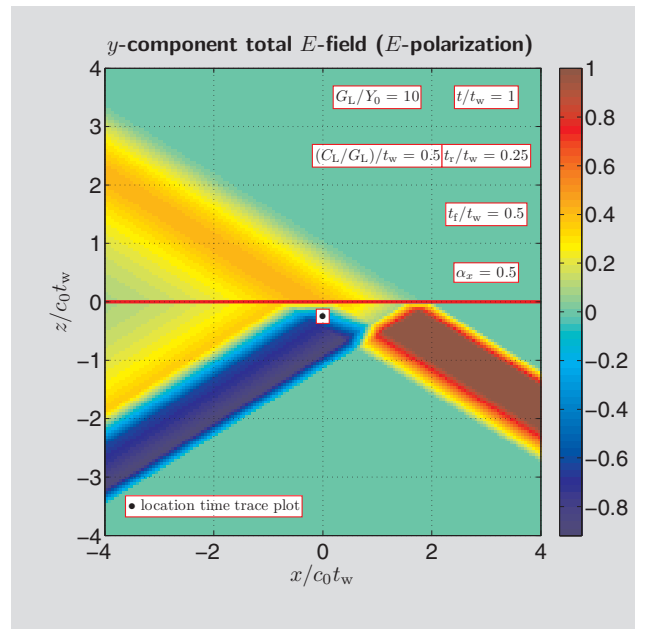

Fig. 5. Density plot in domain about the scattering layer ( $E$-polarization, trapezoidal incident pulse).

transform of $\left.e^{\mathrm{s}}\right|_{\mathrm{ramp}}(t)$ follows, together with $s^{-2}$ as the time Laplace transform of $\operatorname{ramp}(t)$, as

$$
\left.\hat{e}^{\mathrm{S}}\right|_{\mathrm{ramp}}(s)=\hat{S}^{E}(s) \frac{1}{s^{2}}=\left(-1+\frac{T^{E}}{s+\beta^{E}}\right) \frac{1}{s^{2}} .
$$

from which the time-domain pulse shape follows as

$$
\begin{aligned}
\left.e^{\mathrm{s}}\right|_{\mathrm{ramp}}(t)= & \left(-1+\frac{T^{E}}{\beta^{E}}\right) \operatorname{ramp}(t)- \\
& \frac{T^{E}}{\left(\beta^{E}\right)^{2}}\left[1-\exp \left(-\beta^{E} t\right)\right] H(t) .
\end{aligned}
$$

\section{Unit ramp response (H-polarization)}

On account of the results of Section III, the time Laplace transform of $\left.h^{\mathrm{s}}\right|_{\mathrm{ramp}}(t)$ follows, together with $s^{-2}$ as the time Laplace transform of $\operatorname{ramp}(t)$, as

$$
\left.\hat{h}^{\mathrm{s}}\right|_{\mathrm{ramp}}(s)=\hat{S}^{H}(s) \frac{1}{s^{2}}=\left(-1+\frac{T^{H}}{s+\beta^{H}}\right) \frac{1}{s^{2}} .
$$

from which the time-domain pulse shape follows as

$$
\begin{aligned}
\left.h^{\mathrm{s}}\right|_{\mathrm{ramp}}(t)= & \left(-1+\frac{T^{H}}{\beta^{H}}\right) \operatorname{ramp}(t)- \\
& \frac{T^{H}}{\left(\beta^{H}\right)^{2}}\left[1-\exp \left(-\beta^{H} t\right)\right] H(t) .
\end{aligned}
$$

From these results, the pulse shapes $\left\{E_{0}^{\mathrm{r}}, H_{0}^{\mathrm{r}}\right\}(t)=$ $\left\{E_{0}^{\mathrm{r}}, H_{0}^{\mathrm{s}}\right\}(t)$ of the reflected wave in $\mathcal{D}^{-}$and the pulse shapes $\left\{E_{0}^{\mathrm{t}}, H_{0}^{\mathrm{t}}\right\}(t)=\left\{E_{0}^{\mathrm{i}}, H_{0}^{\mathrm{i}}\right\}(t)+\left\{E_{0}^{\mathrm{s}}, H_{0}^{\mathrm{s}}\right\}(t)$ of the transmitted wave in $\mathcal{D}^{+}$can be constructed. Figures 3-6 show results for an incident wave with trapezoidal pulse shape. The figures indicate how drastically the pulse shapes can deviate from the one of the incident wave, as well as how different $E$-polarized field behave from $H$-polarized fields.

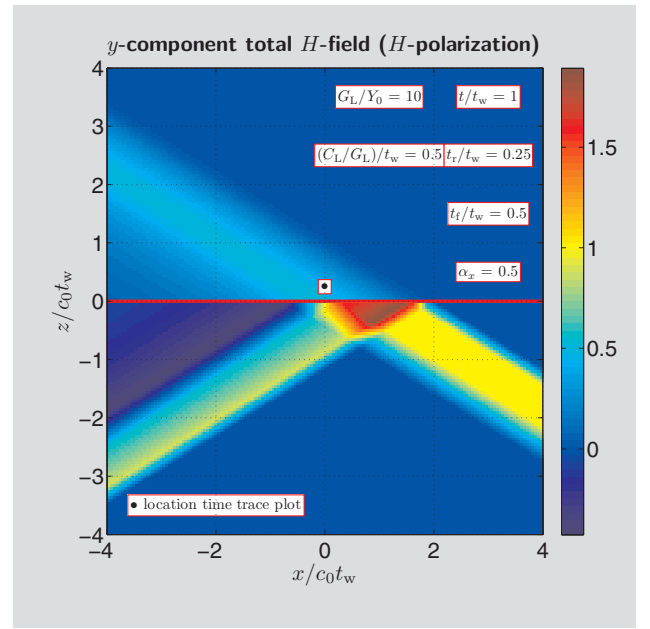

Fig. 6. Density plot in domain about the scattering layer ( $H$-polarization, trapezoidal incident pulse).

\section{CONClusion}

A semi-analytical method is proposed to model the timedomain interaction of an incident, pulsed, electromagnetic field with a thin, high-contrast, finely layered structure via a thin-sheet boundary condition that expresses the in-plane conduction and contrast electric polarization currents in the structure in terms of the exciting incident field. It provides a novel angel to exam the ground plane effects in the complex IC packaging structures.

\section{ACKNOWLEDGMENT}

The authors would like to thank A. Deutsch, B. J. Rubin and J. D. Morsey at IBM T.J. Watson Research Center for many helpful discussions.

\section{REFERENCES}

[1] I. T. Chiang and W. C. Chew, "Thin dielectric sheet simulation by surface integral equation using modified RWG and pulse basis," IEEE Trans. Antennas Propag., vol. 54, no. 7, pp 1927-1934, Jul. 2006.

[2] R. F. Harrington and J. R. Mautz, "An impedance sheet approximation for thin dielectric shells," IEEE Trans. Antennas Propag., vol. 23, pp 531-534, Jul. 1975.

[3] I. S. Koh and K. Sarabandi, "A new approximate solution for scattering by thin dielectric disks of arbitrary size and shape," IEEE Trans. Antennas Propag., vol. 53, no. 6, pp 1920-1926, Jun. 2005.

[4] A. T. De Hoop, Handbook of Radiation and Scattering of Waves, London, Academic Press, 1995.

[5] Rumsey, V. H., "The reaction concept in electromagentic theory", The Physical Review, vol.94, pp. 1483-1491, June 1954.

[6] R. F. Harrington, Time-Harmonic Electromagnetic Fields, New York, McGraw-Hill, 1960.

[7] R. E. Collin, Field Theory of Guided Waves, 2nd ed., New York, IEEE Press, 1991.

[8] T. B. E. Senior, "Approximate boundary conditions", IEEE Trans. Antennas Propag., vol. 29, pp. 826-829, September 1981.

[9] T. B. E. Senior and J. L. Volakis, Approximate boundary conditions in electromagnetics, London, IET Press, 1995.

[10] M. Schoenberg and F. Muir, "A calculus for finely layered anisotropic media," Geophysics, vol.54, no. 5, pp. 581-589, May 1989.

[11] J. M. Hovem, "Acoustic waves in finely layered media", Geophysics, vol.60, no. 4, pp. 1217-1221, July-August 1995. 